\title{
The Effectiveness of the Approach to Integration of Community Leaders in Tuberculosis Detection Chain in Rural Areas
}

\author{
John Baelani Inipavudu ${ }^{1 *}$, John Lusambu Kusa ${ }^{2,3}$, Joel Kafumbi Katsiatsiaa ${ }^{2,4}$, Michael Mitondo Mbuta ${ }^{2,6}$ \\ and Romaine Kiluwa Kambela ${ }^{2,5}$ \\ ${ }^{1}$ Higher Institute of Medical Techniques Marie Queen of Peace Kenge (ISTM-MRP), DRC \\ ${ }^{2}$ Superior Institute of Medical Techniques Marie Queen of Peace Kenge (ISTM-MRP), RDC
}

${ }^{3}$ Provincial Coordination of Leprosy and Kwango Tuberculosis, Kenge Health Zone, RDC

${ }^{4}$ CARAMAL Project (Community Access to Rectal Artesunate for Malaria), Kenge Health Zone

${ }^{5}$ Central Office of the Kenge Health Zone, DRC

${ }^{6}$ TIPTOP project (Transforming Intermittent Preventive Treatment for Optimal Pregnancy), DRC

*Corresponding author: John Baelani Inipavudu, Higher Institute of Medical Techniques Marie Queen of Peace Kenge (ISTM-

MRP), Kenge, DRC

\section{ARTICLE INFO}

Received: 幽 May 06, 2019

Published: 蔧 May 15, 2019

Citation: John Baelani I, John Lusambu K, Joel Kafumbi K, Michael Mitondo M Romaine Kiluwa K. The Effectiveness of the Approach to Integration of Community Leaders in Tuberculosis Detection Chain in Rural Areas. Biomed J Sci \& Tech Res 18(1)-2019. BJSTR. MS.ID.003096.

Abbreviations: DRC: Democratic Republic of Congo; TDSB: Diagnosis and Treatment Health Center; ZS: Health Zone; HGR: General Reference Hospital; TCSC: Tuberculosis Screening and Treatment Center; CSOs: Civil Society Organizations; NGOs: NonGovernmental Organizations; UGB: Basic Management Unit; CS: Health Centers; BCZS: Central Office of the Health Zone

\section{ABSTRACT}

Tuberculosis is a public health problem in Kwango Province . Assessments carried out by a CPLT Kwango (2005-2015) showed the limitations of the health professional-centered approach in detecting TB. this the study is based on the under-detection of tuberculosis observed for more than 10 years in the Kahemba Health Zone. It aimed to determine the effectiveness of the approach of involving community leaders in the detection of tuberculosis. She used a method Comparative quasi- experimental in longitudinal and prospective vision to detect from improvements in TB detection + interventions with the involvement of community leaders (experimental group) vis-à-vis those centered on the health professional (control group). The survey method and the document analysis technique were used to collect the data. Analyzes focused on the comparison test including the chi-square with which the results were compared to two levels: first in the intervention zone at different times and at different areas in the same period. Compared results from two areas revealed a significant increase in detection in the area that integrated the community approach versus the control area ( $90 \%$ vs. 54). The study recommended that health authorities and providers collaborate with community leaders to improve TB + detection .

Keywords: TB + detection; TB messages; Communication; Community leader; Tuberculosis

\section{Introduction}

The World Health Organization estimates that about one-third of estimated TB cases are either undiagnosed or undeclared. It is

often the most marginalized and disadvantaged populations that are affected, for example the poor [1] women, children, migrants, refugees, minors and drug addicts. Even when suspected cases 
are identified, the disease is often diagnosed and treated late. As a result, it causes more damage, and treatment may be more difficult and more expensive. Among the diseases that constitute the major problems of public health in the world, tuberculosis is a disease of poverty and sometimes it is found that health services face problems of geographical, financial and sometimes cultural accessibility for the populations. Services $[2,3]$. Factors that increase the risk of exposure in the community include smoking [4], undernutrition that causes malnutrition. This indeed explains the link between foods and the risk of exposure of TB, which is largely facilitated by a deficiency of immunity [5].

The Democratic Republic of Congo (DRC) is among the countries most affected by this disease. It ranks 9th in the world and 3rd in Africa (National Program for the fight against Leprosy and Tuberculosis DRC, PNLT 2017, Congolese News Agency, 2017, Ngiambukulu, 2017). Current evidence has demonstrated the limitations of the approach to control strictly based on health professionals. Hence, [6] believes that reaching out to isolated populations and improving detection and reporting of TB cases requires new and sustainable approaches that do not stop at the institutional level alone. existing health services, but which address community structures and households. The Ninth Report of the World Health Organization Committee of Experts on Tuberculosis has already supported since 1974 that it was very important to involve the community in the implementation of tuberculosis control programs, through opinion leaders and community-based organizations $[7,8]$.

Tuberculosis control concerns all community groups including primary, secondary and university education institutions, politicoadministrative authorities, religious denominations, NGOs, CBOs, public and private enterprises, community relays, groupings young people, even the entire population (Congolese Press Agency, ACP 2018). The strength of this approach lies in the fact that if the message passes through the groupings within the community, the whole population will be informed and sensitized in less time and at a lower cost. For nearly a decade, the Kahemba Health Zone has become a shadow of its own. Under-detection of TB cases has become the rule rather than the exception. The overall annual rate of detection never exceeds $30 \%$ while the standard set by the NTP requires detection of at least $80 \%$ of expected TB in the population. This worrying situation was an irritation for this study. The approach adopted e was to empower the community in this health area, particularly that of the city Kahemba with five health centers grouped in the Diagnosis and Treatment Health Center (TDSB) General Hospital Reference of Kahemba, through these leaders, to control tuberculosis.

The integration of this community-based approach follows the results achieved during the celebration of World TB Day on March 24, 2016, when the CPLT team organized conferences in the city of Kenge. In addition to this, three high schools and members of the provincial government were sensitized. In the aftermath of these activities, a large crowd came to the office of the CPLT. After consultation, twelve cases of bacteriologically confirmed tuberculosis had been detected in two days. The study conducted in the Kahemba Health Zone was designed to determine the effectiveness of the approach of integrating community leaders into improving the detection of tuberculosis. In particular, it answered the following questions: what is the effectiveness of the approach of integration of community leaders in improving the detection of tuberculosis? Is their contribution significant in guiding patients and detecting TB? To answer these questions, it was first necessary to identify the actors involved in the fight against tuberculosis, namely health service providers and community leaders; second, to demonstrate the contribution of each one in the orientation of patients suspected of having tuberculosis to the screening centers; and finally compare the results obtained in time and space in terms of patient orientation, $\mathrm{TB}+$ detections among the oriented and finally compare the evolution of the detection in the two zones.

\section{Literature Paper}

Tuberculosis is one of the infectious diseases causing the most deaths in the world. It is also a leading cause of death among people living with HIV, accounting for $35 \%$ of deaths among this population in 2015. Tuberculosis and HIV, as well as gender-related challenges and human rights in the fight against these diseases, are therefore closely linked. Tuberculosis is a disease of poverty and inequality. A number of human rights or gender-related factors may hinder the effectiveness, accessibility and sustainability of TB programs and services, as explained in this section. The application of human rights is threatened in the lives of many people living with or at risk of TB. People who face overcrowding, poorly ventilated environments and nutritional deficiencies are vulnerable to the disease and are likely to be at a disadvantage and do not receive reliable information about $\mathrm{TB}$, or no access to good TB services. Although it is possible that these are free, poverty can affect certain factors such as transport to access or a good diet that is conducive to successful treatment [9-11].

Tuberculosis remains a public health problem in sub-Saharan Africa, where health services face problems of geographical, financial and sometimes cultural accessibility for the populations served [12]. The Democratic Republic of Congo (DRC) is among the countries most affected by this disease. It occupies the 9th position in the world and 3rd in Afrique (National Program of Fight against Leprosy and Tuberculosis DRC, NTP 2017, Congolese Press Agency, 2017 Ngiambukulu, 2017). One-third of TB cases are neither diagnosed, treated nor reported. Even in identified patients, TB is often diagnosed and treated late. In order to identify and treat cases that are not, and to intervene at an early stage of the disease, it is necessary to mobilize a wide range of actors already involved in community activities: NGOs and other civil society organizations working in the field. primary health care, HIV, maternal and child health, but have not yet included tuberculosis in their activities [13]. 
Improved screening in rural areas necessarily implies decentralization and the integration of tuberculosis control activities with other primary health care activities. The effective participation of communities in national planning for TB control is neither complex nor difficult to achieve. However, it requires commitment, flexibility and a strong will to build an effective TB response that benefits the entire population. Community involvement enriches the process and adds a special touch in terms of skills, knowledge, diversity and end product integrity that otherwise would not be part of it [6]. There is growing interest in the community's contribution to the provision of care for tuberculosis patients and AIDS patients [14]. Tuberculosis management involves the use of resources outside formal health systems. The involvement of the community through PATB support committees has increased the range of solutions to the problems faced by TB patients and healthcare providers. For community involvement to effectively strengthen the performance of health services, the functional integration of community-based activities into the health care system, a coherent division of tasks among all stakeholders and a balance of power relations between the different contributor's care is essential. Under these conditions, community involvement strengthens the capacity of health centers to better meet the demand for care from chronic patients [7].

\section{Materials and Methods}

\section{Description of the Mi Place of Study}

This study was conducted mainly in the city of Kahemba, located in the capital of the health zone (ZS) of Kahemba. ZS Kahemba is one of fourteen in the Kwango Provincial Tuberculosis Coordination and has four CSDTs. On the organizational level, it has a large city, capital of the ZS and CSDT HGR Kahemba which abounds five health centers. The city of Kahemba accuses some difficulties of drinking water; which is at the base of several diseases like Konzo . Most of the population is peasant; she saw field work and small shops with border traffic with Angola. The intervention area covers a CSDT, in this case, the General Reference Hospital (HGR) , and has a population of 105. 131 inhabitants served by 5 state health areas (Kamayala, Kabongo, Kambanguya, Shamusenge and Kahemba). The control area covers a Tshiweka CSDT with 103371 Inhabitants. Each CSDT contains the same community institutions under the management of the CSDT nurse. The annual number of expected cases of tuberculosis, calculated on the basis of $150 \mathrm{TP}+$ per 100.000 inhabitants according to the PNLT is 158 for the zone of intervention and 155 for the zone control. The two zones do not show obvious socio-cultural disparities and at the level of the annual product per inhabitant even in terms of chosen community leaders.

\section{Sampling Methods and Technique Collection of Donations}

This study is comparative longitudinal in nature prospect. It is based on a quasi-experimental design aimed at identifying improvements resulting from $\mathrm{TB}+$ detection interventions with the involvement of community leaders (Experimental Group) vis-à-vis those focused on the health professional alone (Control Group) . The Tuberculosis Screening and Treatment Center (TCSC) at Kahemba General Referral Hospital (HGR) was considered to be an intervention area (Experimental Group) and CSDT Tshiweka was the control area. A health survey method based on a simple random probability sample and the literature review were used to collect the data used as the basis for the analysis. Community leaders were selected based on an established list of community groupings. The procedure was to identify the first community groups each with its leaders from which a list used for the selection of these was prepared. As a result of this selection , 10 community groups were identified from where 207 community leaders were selected. The size of this sample was calculated by the following Fisher formula (Equation 1):

$$
n=Z^{2} * \frac{p \cdot q}{d^{2}} \text { [Equation 1] }
$$

Or,

$Z$ : the parameter of the normal distribution 5\% signify threshold caution (either $\mathrm{Z}=1.96$ );

$P$ : the probability of selecting a community leader is $25 \%$;

$q=1-p$ : the probability of not being selected as a community leader, representing the proportion of people who have not been elected as leader of community groups , is also $75 \%$;

$d$ : the degree of accuracy of the estimate, is in practice of $75 \%$.

Under these conditions, the size of the " $\mathrm{n}$ " sample was 195 people. A $10 \%$ increase in size has been planned in order to deal with the possibility of non-responses. What brought us a sample of 215 interviewees? And as expected, 8 people unfortunately could not meet all the selection criteria; from which only 207 community leaders were selected.

Regarding TB suspects, two sample $\mathrm{s}$ probabilistic and exhaustive were taken: one in the intervention zone and the other in the control zone. These samples were therefore included all the coughing passed the TDSB to get tested, whether or not directed by community leaders. All subjects were registered in the tuberculosis registry from which we recorded them in their entirety. The size of this sample was 725 for the Kahemba HGR CSDT (Intervention Area) and 259 for the Tshiweka CSDT (Control Zone). The data collected thus cover the period from January to December 2016 and January to December 2017. The respondents were mostly interviewed by appointment, of a ns u r o u community tuberculosis screening and treatment centers. In some cases, are inquest were taken in impromptu, without an appointment was first presided 'action administered a questionnaire or an interview. 
Selection Criteria: The researchers have established the following criteria before including any subject in the study sample:

a. Being male or female living in Kahemba health zone ;

b. Have been trouser during the period of the study;

c. Visiting the CSDT for tuberculosis screening

d. Whether or not guided by a community leader

e. Freely accept to participate in the study; and

f. Having the ability to meet unary interrogate in French or in one of the local languages.

Exclusion Criteria: Was supposed to be excluded from the sample, any subject that dwell outside of a zone Kahemba health during the study period, much less speaking neither French language nor the ng local use.

\section{S Technical Analysis Data}

The collected data were analyzed using computer software MS Excel 2010 and SPSS 17.0. The analyzes were mainly based on descriptive and inferential statistics. The descriptive analysis essentially consisted of the calculation of the frequencies on the basis of the following equation (Equation 2) :

$$
f=\frac{F o}{F A} * 100 \quad[\text { [Equation 2] }
$$

Or,

$f=$ Relative frequency

$\mathrm{FO}=$ Frequency observed

$\mathrm{FA}=$ Expected frequency

The inferential analysis was based on the chi-square test $\left(\mathrm{Khi}^{2}\right.$ or $\square^{2}$ ). This allowed the one hand to verify the effectiveness of the use of services TB C S DT before and after integration of the community leaders' involvement policy in the intervention area. On the other hand, the chi-square test made it possible to compare the results obtained in the group with those of the control group . This inferential analysis tested the null hypothesis that there was no relation between the observed distribution of the use of CST services before the involvement of community leaders in awareness (0) and expected after the integration of community leaders in awareness (E). Thus, the following assumptions were tested as:

a. $\mathrm{H}_{0}: \mathrm{E} \neq \mathrm{O}$, there is a significant difference between the distributions of $\mathrm{E}$ and $\mathrm{O}$

b. $\mathrm{H}_{1}: \mathrm{E}=\mathrm{O}$, there is no significant difference between the distributions of $\mathrm{E}$ and $\mathrm{O}$

From an analytical point of view, the chi-square statistic $\left(\mathrm{Khi}^{2}\right.$ or $\square^{2}$ ) is represented by the following formula ( Equation 3):

$$
\chi_{d d l ; p}^{2}=\sum \frac{(E j-O i)}{\cap \quad \text { (Equation 3) }}
$$

Where,

$E_{j}$, the $\mathrm{j}^{\text {th }}$ expected effective users of C S T services

$O_{i}$, the $\mathrm{i}^{\text {th }}$ observed in the use of C S D T services

$d d l$ : the degree of freedom (equal to $n k$ )

$n$ : the total number of the sample

$k$ : the number of estimated parameters (equal to the number of factors)

$p$ : the significance level of $5 \%$, equal to the probability of failure of $\mathrm{H}_{\mathrm{o}}$

\section{Results of the Analysis \\ Profile of Community Leaders}

Table 1 shows the selection of community leaders involved in the fight against TB as their combinations. He notes that church pastors account for $25.12 \%$; primary school principals $17.39 \%$; heads of politico-administrative offices represent $15.94 \%$; secondary school prefects represent $12.08 \%$; OAC $9,18 \%$; heads of parastatal offices represent $8.21 \%$; the managers of mutual societies represent $4.83 \%$; there is $3.86 \%$ for civil society coordinators and neighborhood leaders respectively; there are $1.45 \%$ for the directors general of higher education and university institutions and finally $0.97 \%$ for the chiefs of the markets (Table 1).

Table 1: Distribution of leaders by community group.

\begin{tabular}{|c|c|c|}
\hline Type of community leader & Staff (n) & Percent (\%) \\
\hline Heads of politico-administrative offices & 33 & 15.94 \\
\hline Heads of parastatal offices & 17 & 8.21 \\
\hline Directors General & 3 & 1.45 \\
\hline School directors & 36 & 17.39 \\
\hline Prefects of schools & 25 & 12.08 \\
\hline Pastors responsible for churches & 52 & 25,12 \\
\hline Organizations in Assize Communautaire & 19 & 9.18 \\
\hline (OAC) & 10 & 4.83 \\
\hline Heads of mutuality's & 2 & 0.97 \\
\hline Market leaders & 8 & 3.86 \\
\hline Civil society coordinators & 8 & 3.86 \\
\hline Heads of neighborhoods & 207 & 100 \\
\hline Total & & \\
\hline
\end{tabular}

Source: Authors (2017)

\section{Involvement of Community Leaders in the Orientation of}

\section{Tuberculosis Patients}

Table 2 is an internal comparison in guiding coughing between 2016 and 2017 the HGR Kahemba. It should first be noted that the table compares the two approaches. On one hand, the traditional 
approach used since time e, where the orientation of coughing to health structures to be tested only noted that the responsibility of health workers and community volunteers. The new approach introduced in 2017 is to involve all community leaders in cough orientation to get tested. The comparison of the results for HGR Kahemba where the approach of community leaders is integrated shows that in 2016 with the old approach, 33 caterers were oriented. On the other hand, the year 2017 gives the new approach to the involvement of community leaders is integrated, 326 coughers were directed to health training by community leaders.

Table 2: Gold mentation suspected TB + in the intervention zone between 2016 and 2017.

\begin{tabular}{|c|c|c|c|}
\hline \multirow{2}{*}{ Type of community leader } & \multicolumn{2}{|c|}{ Number of allegedly oriented } & \multirow{2}{*}{} \\
\cline { 2 - 3 } & $\mathbf{2 0 1 6}$ & $\mathbf{2 0 1 7}$ & \\
\hline Heads of Offices & 0 & 9 & 2.76 \\
\hline Directors General & 0 & 16 & 4.91 \\
\hline School directors & 0 & 22 & 6.75 \\
\hline Prefects of schools & 0 & 55 & 16.87 \\
\hline Responsible Pastors & 0 & 102 & 31.29 \\
\hline OAC & 0 & 38 & 11,66 \\
\hline Heads of mutuality's & 0 & 24 & 7.36 \\
\hline Market leaders & 0 & 22 & 6.75 \\
\hline Civil Society Coordinates & 0 & 18 & 5.52 \\
\hline neighborhoods & 0 & 20 & 6.13 \\
\hline $\begin{array}{c}\text { Total presumed to be } \\
\text { oriented by LC [1] }\end{array}$ & 0 & 326 & $100 \%$ \\
\hline $\begin{array}{c}\text { Total suspected referrals and } \\
\text { health workers [2] }\end{array}$ & 33 & 0 & \\
\hline Total presumed oriented & $33(100 \%)$ & 326 & $100 \%$ \\
\hline
\end{tabular}

Source: Authors (2017).

Table 2 also shows that the proportion of Community grouping suspected of orientation where churches pastors are leading with $31.29 \%$ followed by the prefects of schools with $16.87 \%$. Community-based organizations directed $11.66 \%$ of suspected; For the heads of mutual societies $7.36 \%$; school principals and market leaders each directed $6.75 \%$ of the presumed; the chefs de quartiers oriented $6.13 \%$; the coordinators civil society guided $5.52 \%$ last general managers and heads of offices facing respectively 4.91 and $2.76 \%$. The arson compares the number of presumed oriented at health facilities, demonstrates a significant increase of $987.87 \%$ in 2017 with respect to 2016 in the intervention zone (326 against 33; $\mathrm{p}<0.05$ ) (Table 2).

Table 3 compares the rate of referrals in two different areas during the year 2017. On the side of Gen. al Hospital Kahemba Reference (HGR), intervention area which is integrated the new approach involving community leaders in guiding coughing, and across the TDSB Tshiweka that continues to $r$ uses the traditional approach where only health professionals and community volunteers guide. It is observed in the area of intervention that 326 of 725 or $45 \%$ of coughing were referred by community leaders.
In control zone 98 out of $259,38 \%$ of coughers were referred by community relays and health workers using the traditional approach .

These results clearly indicate that s years the involvement of community leaders in the direction of TB suspected, the area of intervention is the TDSB Kahemba would have had 399 suspected tuberculosis. Thanks to the involvement of the leaders, it is observed in addition of 326 suspected. This shows the contribution of leaders in the orientation of coughs. This result is due to the broad reach of community leaders, which makes it possible to reach a large segment of the population in a reduced time and at a lower cost (Table 3).

Table 3: Rate orientation patients in 2017 between area $\mathrm{s}$ is intervention and control.

\begin{tabular}{|c|c|c|c|c|}
\hline \multirow{2}{*}{ Type of tuberculosis } & \multicolumn{2}{|c|}{ HGR Kahemba } & \multicolumn{2}{c|}{ CSDT Tshiweka } \\
\cline { 2 - 5 } & Effective & $\%$ & Effective & $\%$ \\
\hline Presumed oriented & 326 & 45 & 98 & 38 \\
\hline Presumed unoriented & 399 & 55 & 161 & 62 \\
\hline $\begin{array}{c}\text { Total presumed } \\
\text { tuberculosis }\end{array}$ & 725 & 100 & 259 & 100 \\
\hline
\end{tabular}

Source: Authors (2017).

\section{Effectiveness of Involvement of Community Leaders in}

\section{Approach Detection TB}

Table 4 shows the proportion of TB + among those targeted in both zones in 2017. It shows that 62 sister 142 or $43.66 \%$ of TB patients screened + to HGR Kahemba were referred by community leaders and $56.34 \%$ consulted for themselves s. In the Control Zone , 8 out of 77 or $9.41 \%$ of TB + screened patients were directed by the art health personnel. Without the involvement of community leaders, the number of detected $\mathrm{TB}+$ is 80 but with their involvement their $\mathrm{TB}+62$ more that were added on the 80 for a total of 142 . As can be seen, the contribution of community leaders has increased significantly detection (Table 4).

Table 4: Proportion of TB + among the targeted in 2017.

\begin{tabular}{|c|c|c|c|c|}
\hline \multirow{2}{*}{ Type of tuberculosis } & \multicolumn{2}{|c|}{ HGR Kahemba } & \multicolumn{2}{c|}{ CSDT Tshiweka } \\
\cline { 2 - 5 } & Effective & $\mathbf{\%}$ & Effective & $\mathbf{\%}$ \\
\hline Tracked TB + oriented & 62 & 43.66 & 8 & 9.41 \\
\hline Screened TB + unoriented & 80 & 56.34 & 77 & 90.59 \\
\hline Total TB + detected cases & 142 & 100 & 85 & 100 \\
\hline
\end{tabular}

Source: Authors (2017).

Table 5 compares the effectiveness of the approach involving integrated community leaders to HGR Kahemba the traditional approach still used at TDSB Tshiweka. The results show that HGR Kahemba achieved a 90\% detection rate while at TDSB Tshiweka c e rate is $55 \%$. In view of these results, we find that HGR Kahemba has reached the national standard e set by the National Program against Tuberculosis which consists of screening at least $80 \%$ of 
expected cases . On the other hand, CSDT Tshiweka did not reach this norm. The approach is effective especially since it contributed to nearly $44 \%$ in the detection because without this contribution, detection in the intervention zone would remain at $56 \%$ (Table 5).

Table 5: Comparison of the effectiveness of $s$ approach $s$ in the detection of TB + en 2017.

\begin{tabular}{|c|c|c|c|c|c|c|}
\hline \multirow{2}{*}{ Rate of deposition } & \multicolumn{3}{|c|}{ HGR Kahemba } & \multicolumn{3}{c|}{ CSDT Tshiweka } \\
\cline { 2 - 7 } & ATT & F. O & \% & ATT & F. 0 & \% \\
\hline $\begin{array}{c}\text { Total TB + detected } \\
\text { cases }\end{array}$ & 158 & 142 & 90 & 155 & 85 & 55 \\
\hline
\end{tabular}

Source: Authors (2017).

Table 6 shows the change detection in both areas from 2016 to 2017. It should be noted that by 2016 the two areas used the same approach, which is the traditional approach where only health workers were involved in guiding patients. In 2017, however, the HGR intervention area benefited from the involvement of community leaders. For the control area, the screening rate in 2016 was $28.75 \%$ and in the control area it was $21.19 \%$. In 2017, the intervention zone reached the detection rate of $90 \%$ but in the control zone this detection remained at $55 \%$. It should be noted that these two areas were in a particular context. For more than ten years with the conventional approach, no structure has reached a screening rate of at least $80 \%$ as a national standard. It's a great performance for the first time in over 10 years to see that e HGR Kahemba TDSB has tracked over $80 \%$ and reach the following standard to the introduction of the approach community leaders (Table 6).

Table 6: Comparison of the TB + detection rate between 2016 and 2017 in both Zones.

\begin{tabular}{|c|c|c|c|c|c|c|c|c|c|c|c|c|}
\hline \multirow{3}{*}{ Comparison detection } & \multicolumn{6}{|c|}{ HGR } & \multicolumn{6}{|c|}{ CSDT TSHIWEKA } \\
\hline & \multicolumn{3}{|c|}{2016} & \multicolumn{3}{|c|}{2017} & \multicolumn{3}{|c|}{2016} & \multicolumn{3}{|c|}{2016} \\
\hline & ATT & F. 0 & $\%$ & ATT & FO & $\%$ & ATT & FO & $\%$ & ATT & $\begin{array}{l}\text { F. } \\
\text { O }\end{array}$ & $\%$ \\
\hline Total & 153 & 44 & 28.75 & 158 & 142 & 90 & 151 & 32 & 21,19 & 155 & 85 & 55 \\
\hline
\end{tabular}

Source: Authors (2017).

\section{Discussion of Results}

\section{Discussion on the Profile of Community Leaders}

The composition of the different community groups that have integrated the tuberculosis control activities, to which these leaders come, shows that the vast majority of the different segments of the population are represented and that if we work with all these leaders, there is possibility of reaching a large mass of the population. The establishment of this structure emanates from the WHO recommendations, which since 2012 have already requested the involvement of NGOs and Civil Society Organizations (CSOs) in activities to fight against tuberculosis, a policy called "ENGAGE-TB". These recommendations are based on the observation that onethird of estimated TB cases are either undiagnosed or undeclared. It is often the most marginalized and disadvantaged populations that are affected, for example the poor, women, children, migrants, refugees, minors and drug addicts. Even when suspected cases are identified, the disease is often diagnosed and treated late. As a result, it causes more damage, and the treatment can be more difficult and more expensive.

In order to reach isolated populations and to improve the detection and reporting of TB cases, new and sustainable approaches are needed that do not stop at the level of existing health facilities but also address community structures. and households. The Global Fund has been supporting community responses to the three diseases (malaria, HIV and TB) for years. If, given their nature, the formal sector is in the best position to implement elements of the response to HIV and AIDS, TB and malaria, we encourage countries to also seek to address from the bottom up, namely by working with, through and within communities. The Global Fund encourages and supports them to contribute to advocacy efforts, to monitor program effectiveness by holding managers accountable, to program development, service delivery, grant governance at the global level. countries and the governance of the Global Fund. Communities are not just recipients of services. They are also actors whose contribution to the improvement of health is vital. Thus, as communities participate, programs gain a greater impact. They expand the reach of services, involve people in health care, and overcome social rejection and discrimination [15].

\section{Discussion on the Involvement of Community Leaders}

The second table of our study evaluates the impact of the involvement of community leaders in the orientation of the suspected tuberculous (coughing). The results of the study made an internal comparison with those obtained one year before the introduction of this approach in the intervention zone. It appears from this study that in 2016, only the conventional approach was used. The e health personnel were the one to be involved in the referral of patients to health facilities. And as a result, only 33 coughers were referred to the CDST . By cones 2017 , lords we opted for the involvement of community leaders in TB activities , this figure is eleven to 326 patients. The comparison in the MPs of presumptive referrals within the same health facility shows a significant increase of $987.87 \%$ in 2017 compared to 2016 in the intervention zone (326 vs. 33, $\mathrm{p}<0.05$ ).

This increase is explained by the fact that the community at all levels has had at least the opportunity to hear about tuberculosis, but especially the permanent possibility of being oriented towards the health structure in case of cough. Every leader has around him a multitude of people. As soon as he starts the message, each member 
of his community appropriates and becomes in turn a coin into the $\mathrm{c}$ dissemination of hate like that a lot will be notified and will benefit from these services. One of the most important services of this community involvement is to direct the coughers to the health facilities for detection so that the result can determine what to do next. The results obtained corroborate with Drabo et al. [7] who, in his study of community involvement in the care of tuberculosis patients in three health districts in Burkina Faso in 2009 had found that in 12 months (January to December 2006), community involvement has helped to significantly increase the detection of $\mathrm{TMP}+$ in the intervention zone.

These results also allow us to assert the allegations of WHO, which supports non-governmental organizations (NGOs) and other civil society organizations (CSOs) often have effective means of reaching out to vulnerable and isolated population groups. They also allow us to say that If this approach is to be applied in all structures, it is promising and carries a breath of hope in favor of patients lost in the wild and remained undetected for a long time. It also responds to the concerns of WHO (2015) that implementation and expansion of community-based TB control activities remains inadequate despite the obvious need. Implementing this approach will improve the situation. The on the Stop TB Strategy Studies have shown that the community plays an important role in the control of tuberculosis [16].

The results of the Table 3 got down to comparing $r$ the rate of patient orientation in two areas of study during the year 2017. On the side of the General Hospital Kahemba Reference (HGR) , intervention area, and on the other side the CSDT Tshiweka control area. In the intervention area, 326 out of 725 or $45 \%$ of the coughers were directed by community leaders. In control zone 98 out of $259,38 \%$ of the patients were referred by health personnel using the traditional approach. The lie logically, as we can see if the leader Community had not been involved, HGR Kahemba could only have 399 presumed. The involvement of community leaders is an added value. This comparison makes it possible to demonstrate the appropriation of this policy by the population and that its implication makes it possible to increase the rate of orientation with probability of increasing the detection. Although applied for the first time, this approach already shows its effectiveness compared to the old approach which is the classic approach.

By mid-indicators proposed by the WHO to assess the commitment of the community in the fight against tuberculosis, the e first is the definition of referral and notification of new cases : number of new patients tuberculosis (regardless of the form of the disease) that were referred by community health workers and community volunteers, expressed as a percentage of all new tuberculosis patients notified to the basic management unit (UGB) during a given period. As can be seen, the results of the study clearly show that $45 \%$ of the patients arriving at the HGR Kahemba which the intervention zone is were community-oriented whereas in the control zone with conventional approach, the Health workers only referred $38 \%$.

\section{Effectiveness of News has Approach Compared to the Traditional Approach to Detection TB +}

The results of Table 4 showed the proportion of $\mathrm{TB}+$ among the targeted in both zones in 2017. The observation notes that 62 of 142 or $43.66 \%$ of TB + patients diagnosed with HGR Kahemba were referred by the leaders. Community and $56.34 \%$ consulted for themselves. In the control area where the traditional approach is still used , 8 of 77 or $9.41 \%$ of TB patients die + tracked were directed by agent's health. As can be seen, community involvement in active research and cursor orientation in health facilities has increased significantly the detection in the area of speech by providing 62 TB more, which would not be possible without this contribution because conventionally the HGR Kahemba would have had only 80 TB + .

Available evidence demonstrates that when interventions are used in high-burden settings, active case-finding approaches may increase detection of TB cases in the short-term in moderate and high prevalence settings. However, the available evidence does not indicate whether active case-finding interventions can improve treatment success and reduce failure, mortality, and failure of TB treatment [17]. The results presented in Table 5 compared the detection rate in the area of the approach involving community leaders integrated into the HGR Kahemba with that of the traditional approach still used at CSDT Tshiweka. The results show that the Kahemba HGR has reached a detection rate of $90 \%$ whereas at CSDT Tshiweka this rate is $55 \%$. The involvement of community leaders contributed to nearly $44 \%$ in detection. In this contribution, detection in the intervention zone would remain at $56 \%$. Given these results, we find that the HGR Kahemba reached the national standard set by the National Program of Fight Against Tuberculosis which is to detect at least $80 \%$ of expected cases while the TDSB Tshiweka with its classic approach $\mathrm{n}$ has not reached this standard continues to sink into under-detection.

[18] notes that, after having steadily increased until 2006, the number of reported cases is stagnating today, and many of them have been reported late? Prevalence surveys also show that many countries bear a high hidden burden of asymptomatic cases. These elements show the limitations of the current methods of case detection. Much remains to be done to build strong collaboration with civil society organizations, communities and people living with TB to stimulate demand for TB care and make it available to all who need it. [19] notes during the Franco Lao Pneumology Days that the stagnation of detection in 2013 in Lao was linked to the weak involvement of local authorities, health committees and communities in detection. Involving village leaders, health committee members and villagers and community partners is one way to increase testing.

In Table 6 , it was a question of comparing $r$ the development of detection in both areas from 2016 to 2017. It should be noted that by 2016 the two areas using the same approach, which is the traditional approach where only health workers were involved in 
guiding patients. In 2017, however, the HGR incorporated for the first time the approach of involving community leaders. For HGR Kahemba, the detection rate in 2016 was $28.75 \%$ and at CSDT Tshiweka it was $21.19 \%$. In 2017, the area of intervention (HGR Kahemba) reached the detection rate of $90 \%$ but in the control zone ( CSDT Tshiweka) this detection remained at 54.83. It should be noted that these two areas were in a particular context. For more than ten years using the conventional approach, none of the two has achieved a minimum detection rate of at least $80 \%$ as a national standard. They both went under-detection, which generally was around $30 \%$. It's a great performance for the first time in more than 10 years to see that HGR Kahemba has screened over $80 \%$ and reach the norm after the introduction of the approach involving community leaders.

This performance proves that if all communities could actively engage in the fight against this disease by looking for cases lost in the wild and reaching all segments of the population, especially the most marginalized, case detection will increase significantly and this may be one of the pathways towards the goal of eliminating tuberculosis, which WHO has advocated for years. This performance can also help the political and health authorities to set on foot a clear policy of partnership with the community in the fight against tuberculosis , which can help reduce this disease. Measuring performance gives policy makers a golden opportunity to improve health systems and make them more accountable. One of the important uses of performance measurement is to provide clinical practitioners with a record of their actions and a comparison with those of their colleagues. Performance measurement systems should be observed frequently to ensure that they are linked to other mechanisms of health systems and to identify areas for improvement. Experiments are underway to study how performance measurement can be used in Combining with explicit financial incentives to reward the performance of healthcare providers is a promising area for policy and a priority for future research [20].

The Global Plan stresses that the objectives of the Eliminate Tuberculosis program will not be achieved if current efforts continue without a paradigm shift. Of the eight areas where a paradigm shift is needed, the fourth is a community and patient- centered approach. The Global Plan requires additional and important efforts for real engagement and collaboration with key populations, people living with TB, civil society and the private sector. The plan presents an approach to identify, reach and work with key populations. Civil society and community-based organizations must play a key role in planning and delivering TB care through advocacy; actively seeking cases; improving access to care; encouraging accession; providing psychosocial support and reducing stigma; setting up monitoring programs; and facilitating community engagement in research and development [21-26].

\section{Conclusion}

The utter against tuberculosis is a challenge and should not be considered as an issue of are medical professionals only . This study, which was based on WHO recommendations summary E by $\mathrm{E}$ approach engage-TB, has evaluated the effectiveness of the approach involving the art community leaders in the detection of TB. The results obtained make it possible to affirm that the involvement of community leaders in the fight against tuberculosis to control tuberculosis. If properly applied, it significantly improves detection by systematically targeting coughers to health facilities for screening and management. One of the strengths of this approach is that it reaches all levels of society in less time and at a lower cost. The results clearly show that there has been improvement in the detection rate in the intervention area compared to the year before compared to that of the intervention but also compared to the area control (Table 6).

\section{Recommendations}

In view of the results obtained in this research, it is strongly recommended to the peripheral level health authorities, in particular the Central Office of the Health Zone (BCZS) and the Health Centers (CS) to be able to disseminate the community approach in the fight against tuberculosis. To do this, they should encourage associations to broad community-based to integrate TB + messages to other fight against TB activities in their daily activities. Finally, encourage the sponsorship of tuberculosis patients and the creation of association of former tuberculosis patients who can well drain the others to consult the CS.

\section{References}

1. Emmanuelli J, Grosset (2003) Tuberculosis and Poverty/ Journal of Respiratory Diseases 20(2): 169-171.

2. (2018) The fight against tuberculosis requires a commitment from the entire community. Congolese Press Agency, ACP.

3. (2017) DRC among the countries most affected by tuberculosis in the world. Congolese News Agency, ACP.

4. (2009) Tuberculosis \& Tobacco: World Health Organization, Geneva.

5. (2013) Micronutrient supplementation in people with active TB: World Health Organization, Geneva.

6. (2015) The global plan to eliminate tuberculosis. Stop TB Partnership: The paradigm shifts 2016-2020. Geneva, Switzerland.

7. Drabo M, Zerbo R, Berthe A, Ouedrago L, Konfe S, et al. (2009) Community Involvement in TB Care in 3 Health Districts in Burkina Faso." Public Health 21(5): 485-497.

8. Flower St J M (2006) For eradication of tuberculosis, Public health/ Tuberculosis, Le Nouvelliste.

9. (2017) Community systems and responses. Global Fund, Geneva, Switzerland.

10. (2018) Mobilize NGOs and civil society around TB control: World Health Organization, Geneva.

11. Mburu G, Richardson D (2013) Community-based TB and HIV integration. Good practice guide. International HIV/AIDS Alliance and PATH: Hove.

12. Boulahbal F, Chaulet $P$ (2004) Tuberculosis in Africa. Epidemiology and control measures. Med Trop (64): 224-228.

13. (2018 b) Tuberculosis Aide-memoire, January 2018: World Health Organization, Geneva.

14. Maher D, Van Gorkom JLC, Gondrie PCFM, Raviglione M (1999) Community contribution to tuberculosis management in countries with 
high TB prevalence: past, present and future. Int J Tuberc Lung Say 3(9) 762-768.

15. (2017) Technical information note. Tuberculosis, gender and human rights. Global Fund, Geneva, Switzerland.

16. (2014) Annual Tuberculosis Report, Kinshasa. PNLT: DR Congo.

17. Noeske J, Yakam NA, Defoe AJL (2016) Epidemiology of tuberculosis in Cameroon as mirrored in notification data, 2006-2014. Int J Tuberc Lung Say 20(11): 1489-1494.

18. (2013) Global strategy and targets for TB prevention, care and post2015 control: World Health Organization, Geneva, USA.

19. Vangvichit P (2014) National Tuberculosis Control Program in Laos. Days Franco Lao tires ology.

20. Smith PC, Mossialos E, Papanicolas I, Sheila Leatherman (2008) Performance measurement for health system improvement: experiences, challenges and perspectives. WHO European Ministeria

\section{ISSN: 2574-1241}

DOI: 10.26717/BJSTR.2019.18.003096

John Baelani Inipavudu. Biomed J Sci \& Tech Res

(C) This work is licensed under Creative

Submission Link: https://biomedres.us/submit-manuscript.php
Conference on Health Systems: Health Systems, Health and Prosperity. Tallinn (Estonia)

21. M boussa J, Simonnet F, Lefebvre SMC, Card JSI, Yokolo D, et al. (1999) Organization of tuberculosis control in urban and rural areas: Congo experiment. Medicine of Black Africa: 46(8/9).

22. N Giambukulu OD (2017) DRC among the countries most affected by tuberculosis in the world.

23. (2014) Significant Involvement of Tuberculosis Control Communities in National Planning. Halte Partnership on Tuberculosis.

24. (2012) ENGAGE TB: World Health Organization, Geneva.

25. (2014) Report of tuberculosis in the world: World Health Organization, Geneva.

26. (2012) Tuberculosis and TB / HIV Advocacy Guide, Kinshasa. PNLT: DR Congo.

$\begin{array}{ll}\text { BIOMEDICAL } & \text { Assets of Publishing with us } \\ \text { RESEARCHES } & \text { - Global archiving of articles } \\ & \text { - Immediate, unrestricted online access } \\ & \text { - Rigorous Peer Review Process } \\ \end{array}$

